\title{
Pattern of peripheral neuropathy in systemic lupus erythematosus: clinical, electrophysiological, and laboratory properties and their association with disease activity Mohamed Hassan Imam ${ }^{\mathrm{a}}$, HamdyKhamis Koriem ${ }^{\mathrm{a}}$, Marwa Mohamed Hassan ${ }^{\mathrm{a}}$, Abeer Shawky El-Hadidi ${ }^{\text {, }}$, Niveen Abdallah Ibrahim ${ }^{a}$
}

aDepartment of Physical Medicine, Rheumatology and Rehabilitation Clinic, Faculty of Medicine, Alexandria University, Egypt, bepartment of Clinical and Chemical Pathology, Faculty of Medicine, Alexandria University, Egypt

Correspondence to Dr. Niveen Abdallah Ibrahim Ahmed, MBBCh, Master of Physical Medicine, Rheumatology and Rehabilitation, Department of Physical Medicine, Faculty of Medicine, Alexandria University, Egypt.

Tel: +201223797072

e-mail: niveenabbdallah@gmail.com

Received 27 March 2019

Accepted 17 June 2019

Egyptian Rheumatology \& Rehabilitation 2019, 46:285-298

\begin{abstract}
Aim
To study clinical, electrophysiological, and laboratory properties of peripheral neuropathy (PN) in systemic lupus erythematosus (SLE) and their association with disease activity.

Patients and methods

A total of 30 patients who met the American College of Rheumatology case definition criteria for SLE-PN and 30 age-matched and sex-matched patients with SLE without PN were selected from the Main Alexandria University Hospital Physical Medicine, Rheumatology and Rehabilitation clinic. Demographic data, SLE-related clinical, laboratory data, Systemic Lupus Activity Measure (SLAM) index, and nerve conduction studies were done. This case-control study compared clinical and SLE-related features, laboratory, and SLAM index in patients with SLE with PN versus those without neuropathy.
\end{abstract}

\section{Results}

The results showed that the most common PN subtype was sensorimotor polyneuropathy which occurred in $18(60 \%)$ patients; the most common PN pathology was axonal degeneration, which occurred 19 (63.3\%) patients; and the most common associated nerve entrapment was carpal tunnel syndrome in 10 (33.3\%) patients. In comparison between group I (SLE with PN) and group II (SLE without PN), there was no statistically significant difference between the two groups regarding demographic data, disease duration, and lupus clinical features, except malar rash and lupus nephritis, which showed significant increase in patients with SLE with PN compared with patients with SLE without PN $(P=0.003$ and $P<0.001$, respectively). There was no statistically significant difference among $P N$ subtype groups regarding sex, age, and immunological markers. Regarding diseases activity, SLAM index showed a significant increase in patients with SLE with PN compared with patients with SLE without PN $(P=0.006)$.

\section{Conclusion}

The pattern of neuropathy in SLE is mainly axonal. Moreover, the most common PN subtype is sensorimotor polyneuropathy. The study suggests significant association of PN in patients with SLE with nephritis, malar rash, and SLAM index.

\section{Keywords: \\ Egypt Rheumatol Rehabil 46:285-298 \\ () 2019 Egyptian Society for Rheumatology and Rehabilitation 1110-161X}

disease activity, peripheral neuropathy, systemic lupus erythematosus

\section{Introduction}

Systemic lupus erythematosus (SLE) is a multisystem autoimmune disorder with wide scope of clinical introductions. It affects both the central nervous system (CNS) and the peripheral nervous system (PNS). The American College of Rheumatology proposed case definitions and classification criteria for 19 CNS and PNS syndromes observed in SLE [1]. Peripheral neuropathy (PN) is a perceived manifestation of SLE [1-5]. However, unique sorts of neuropathy happen in SLE, including symmetrical polyneuropathy, mononeuropathy, and cranial neuropathy $[1,3,5,6]$; in addition, other dysimmune neuropathies, including (GBS), chronic inflammatory demyelinating polyneuropathy (CIDP), as well as neuropathies owing to non-SLE causes, for example, nerve entanglement or other related clutters, are additionally found in patients with SLE $[7,8]$. In perspective on this heterogeneity, it is obvious that the revealed commonness of neuropathy in SLE has been variable $[9,10]$. However, because of contrasts in the criteria used to characterize neuropathy, regardless of whether clinical or electrophysiological, studies that have fundamentally utilized electrophysiological criteria for neuropathy have frequently revealed higher prevalence

\footnotetext{
This is an open access journal, and articles are distributed under the terms of the Creative Commons Attribution-NonCommercial-ShareAlike 4.0 License, which allows others to remix, tweak, and build upon the work non-commercially, as long as appropriate credit is given and the new creations are licensed under the identical terms.
} 
rates as they incorporate various asymptomatic patients with nerve conduction abnormalities $[9,10]$. The clinical presentation of PN relies upon the diameter of the affected nerve, the sort of demyelinating or axonal lesions, and their acute or chronic occurrence [11]. Routine nerve conduction studies just mirror the activity of the fast conducting myelinated A nerve fibers, which are physiologically irrelevant to pain. The overwhelming neuropathic manifestations are related to abnormalities of other smaller nerve fibers. Hence, quantitative sensory testing can evaluate small nerve fiber function [12], which should be subsequently applied to patients with SLE-related PNs.

The pathogenesis of SLE-related neuropathy is obscure, and the few pathological studies of the peripheral nerves in SLE have revealed axonal degeneration, inflammatory changes, and vasculitis [13]. Typical vasculitic neuropathy, because of medium-sized vessel vasculitis, is uncommon in SLE, and this may propose the likelihood of various pathogenetic components for the different types of SLE-related neuropathy [14]. A lot of mechanisms have been cited to explain the peripheral damage in lupus. Neurogenic inflammation is interceded by the release of different neuropeptides such as calcitonin gene-related protein, substance $\mathrm{P}$, nitric oxide and chemokines resulting in vasodilatation, increasing vascular porousness and cell trafficking. The major inflammatory mediators released from immune cells act on sensory neurons, inducing peripheral sensitization and hyperalgesic phenomena. In addition, after damage, this natural inflammatory response could encourage the pathogenetic activity of antineural autoantibodies, in addition to ischemic vascular mechanism, by vasa nervorum vascularitis or by microthrombi linked to antiphospholipid antibodies. The other legitimate mechanisms are immunologic cause by a direct aggression by antibodies, entraining obliteration of the peripheral nerve component [15-17].

In the literature, research studies on the PNS manifestations are mostly represented by case reports or case series with few patients [18]. Furthermore, the PN has not been well prescribed in SLE in terms of onset, severity, clinical associations, and electrophysiological characteristics. Therefore, more studies are necessary to characterize PN in SLE with respect to the patient's clinical lupus properties, serologic markers, disease activity, and electrophysiological data.

\section{Patients and methods}

All patients were selected from those attending the Outpatient Clinic of Physical Medicine, Rheumatology and Rehabilitation Department and Rheumatology Outpatient Clinic, Internal Medicine Department, The Main Alexandria University Hospital. They were divided into two groups:

Group I (SLE with PN) included 30 adult patients with SLE fulfilling the Systemic Lupus International Collaborating Clinics criteria [19]; definitions of PNs were provided by the American college of rheumatology (ACR) nomenclature and case definitions for neuropsychiatric syndromes in patients with SLE [1].

The following were the clinically exclusion criteria:

(1) Patients aged less than 18 years.

(2) Patients with connective tissue diseases.

(3) Patients with other overt causes of PN such as follows:

(a) Endocrinal: for example, diabetes mellitus and hypothyroidism.

(b) Metabolic: such as renal and liver failure.

(c) Drugs and toxins: for example, alcohol abuse, vincristine, metronidazole, phenytoin, isoniazid, and fluoroquinolone.

(d) Compression neuropathy.

(e) Traumatic neuropathy.

(f) Infection: Herpes zoster and leprosy.

(g) Malignancy.

Group II (SLE without PN) included 30 adult patients with SLE fulfilling the Systemic Lupus International Collaborating Clinics criteria [19] without clinical manifestations of PN and confirmed by normal electrophysiological study.

The two groups were subjected to the following:

(1) Recording of historical data for patients including personal, present, past, family, drug and obstetrical history.

(2) Registration of anthropometric measures.

(3) Thorough clinical examination including neurological and musculoskeletal examination.

(4) Cold stress test as a quantitative sensory test, which evaluate $\mathrm{A} \delta$ and $\mathrm{C}$ fibers [20].

(5) Systemic Lupus Activity Measure (SLAM) index.

(6) Electrophysiological assessment including motor conduction study of median, ulnar, tibial and common peroneal nerves at both sides. Sensory conduction study of (median, ulnar and sural) nerves at both sides. Bilateral blink reflex and sympathetic skin response test was examined in both hands.

(7) Laboratory assessment including complete blood count (CBC), erythrocytes sedimentation rate 
(ESR), C-reactive protein (CRP), serum creatinine, urea, complete urine analysis, antinuclear antibodies (ANA), anti-ds DNA, C3, and C4.

\section{Statistical analysis}

Data were fed to the computer and analyzed using IBM SPSS software package version 20.0. (IBM Corp., Armonk, New York, USA). Qualitative data were described using number and percent. The Kolmogorov-Smirnov test was used to verify the normality of distribution. Description of the quantitative data was done using the mean $\pm \mathrm{SD}$ in normally distributed data, whereas using the median (the minimum and the maximum) in not normally distributed one. Significance of the obtained results was judged at the 5\% level. Comparison between different groups regarding categorical variables was tested using $\chi^{2}$-test, and when more than $20 \%$ of the cells have expected count less than 5 , correction for $\chi^{2}$ was conducted using Fisher's exact test. Student $t$-test was used for normally distributed quantitative variables, to compare between two studied groups, whereas Mann-Whitney test was used for abnormally distributed quantitative variables. Distributions of the different electrophysiological parameters were calculated at 5th and 95th percentiles. Nerve conduction parameters were considered abnormal if they were above the 95th percentile of reference values for latency and below the 5th percentile for amplitude and velocity. Electrophysiological laboratory reference values were previously obtained from 100 healthy persons

\section{Results}

(1) The frequency of PN in the selected SLE patients sample was 39.4\%; the recruitment of patients with SLE with PN required examination of 76 patients with SLE.

(2) Three (10\%) patients developed PN simultaneously with SLE, and the remaining 27 (90\%) patients developed PN 30.933 \pm 39.34 months after the onset of SLE.

(3) Sensation was normal in $5 / 30(16.6 \%)$ patients in group I, whereas 25/30 (83.3\%) patients showed abnormal sensory manifestations. Pain was reported in five $(16.6 \%)$ patients, numbness or paresthesia was reported in $15(50 \%)$ patients, glove and stocking hypoesthesia was presented in $12(40 \%)$ patients, whereas only two (6.6\%) patients had peripheral anesthesia. Sensory complaints were present in both upper limb (ULs) and lower limb (LLs) in 14 (46.7\%) patients, whereas four (13.3\%) patients complained of abnormal sensation in lower limbs only.
(4) The most common PN-related symptoms were numbness and weakness in affected regions in $20(66.6 \%)$ patients. Five (16.6\%) patients experienced only pain in the affected regions, gait unsteadiness was reported by three (10\%) patients, four (13.33\%) patients experienced postural hypotension and changes in sweating pattern, whereas only one (3.3\%) patient was confirmed to have PN according to the electrophysiological tests, although no clinical symptoms of PN were observed.

(5) Abnormal neuropathy signs were found in $29 / 30$ (96.6\%) patients. Reduced pain sensation was seen in 19 (63.3\%) patients: 14 bilateral and five unilateral. Two (6.6\%) patients with reduced pain sensation also had bilateral loss of vibration sense. Ankle reflexes were absent in 20 (66.6\%) patients, of whom 16 (53.3\%) were bilateral. Distal muscle weakness was present in five (16.6\%) patients, affecting predominantly lower limbs. Four (13.3\%) patients had generalized muscle wasting. Two patients with evidence of transverse myelitis had lower limbs proximal and distal muscle weakness.

(6) Sixteen (53\%) patients were symmetrically affected by $\mathrm{PN}$, whereas 14 (47\%) patients had asymmetrical PN pattern, which involved the sensory (42\%) and motor nerves (57\%).

(7) The cranial neuropathy cases in the current study included two male patients who presented with trigeminal neuropathy and two female patients with features of old facial palsy presented by synkinesis despite normal electrophysiological study result.

(8) Regarding ANA immunofluorescent patterns, 18 (30\%) patients had speckled pattern, six (10\%) patients had the homogenous pattern, two (3.33\%) patients had homogenous and rim pattern, another two (3.33\%) patients had cytoplasmic pattern, one (1.66\%) patient had rim pattern, another one (1.66\%) patient had a fine granular pattern, one (1.66\%) patient had mixed fine granular and speckled pattern, 15 (25\%) patients had weak positive ANA titer equal to $1 / 40$ without specific pattern, whereas 14 (23.3\%) patients were ANA negative.

(9) Skin sympathetic response (SSR) showed normal values in 17/30 (56.6\%) patients of group patients (SLE with PN), whereas it showed abnormality in $13(43.33 \%)$ patients of the same group [it was unobtainable in seven (23.33\%) patients, and it showed delayed latency in six (20\%) patients; four of those patients with abnormal SSR showed clinical manifestations of autonomic neuropathy, 
whereas the remaining nine patients had not any manifestations of autonomic neuropathy; thus, they might have had subclinical autonomic dysfunction].

\section{Distribution of the studied cases according to} subtypes of peripheral neuropathy in systemic lupus erythematosus with peripheral neuropathy (group I) ( $n=\mathbf{3 8}$ conditions in $\mathbf{3 0}$ patients)

Eighteen (60\%) patients had sensorimotor polyneuropathy (the most common subtype), whereas none of the patients had acute inflammatory

Table 1 Distribution of the studied cases according to subtypes of peripheral neuropathy in group I ( $n=38$ conditions in $\mathbf{3 0}$ patients)

\begin{tabular}{lc}
\hline Subtypes of peripheral neuropathy & $N(\%)$ \\
\hline Sensory polyneuropathy & $1(3.3)$ \\
Sensorimotor polyneuropathy & 18 \\
& $(60.0)$ \\
Noncompression mononeuropathy single & $2(6.7)$ \\
Mononeuritis multiplex & 7 \\
& $(23.3)$ \\
Cranial neuropathy & 4 \\
& $(13.3)$ \\
Length-dependent small fiber neuropathy & $1(3.3)$ \\
Non-length-dependent small-fiber neuropathy & $1(3.3)$ \\
Autonomic neuropathy & 4 \\
& $(13.3)$ \\
Acute inflammatory demyelinating polyneuropathy & 0 \\
(Guillain-Barre syndrome) & \\
Chronic inflammatory demyelinating polyneuropathy & 0 \\
Plexopathy & 0 \\
\hline
\end{tabular}

demyelinating polyneuropathy (Guillain-Barre syndrome) or CIDP or plexopathy (Table 1 and Fig. 1).

\section{Distribution of the studied cases according to} pathology of peripheral neuropathy in group I $(n=30)$

Nineteen (63.3\%) patients had axonal degeneration (the most common type), one (3.33\%) patient had demyelination, eight (26.6\%) patients had mixed pathology (demyelination and axonal degeneration), and two (6.7\%) patients had pure small fiber neuropathy (SFN) (Table 2 and Fig. 2).

\section{Distribution of the studied cases according to associated nerve entrapments in group I ( $n=32$ conditions in $\mathbf{3 0}$ patients)}

Regarding common entrapments, 10 (33.3\%) patients had carpal tunnel syndrome (CTS) (the most common entrapments), whereas 14 (46.66\%) patients showed no evidence of entrapments (Table 3 and Fig. 3).

\section{Comparison among peripheral neuropathy subtypes} regarding demographic data (sex and age) in group I There was no statistically significant difference among PN subtypes regarding sex and age (Table 4).

\section{Comparison among peripheral neuropathy subtypes regarding immunological markers in group I}

There was no statistically significant difference among PN subtype groups regarding ANA, APLs, C3, C4, anti-dsDNA, and MMP9 (Table 5).

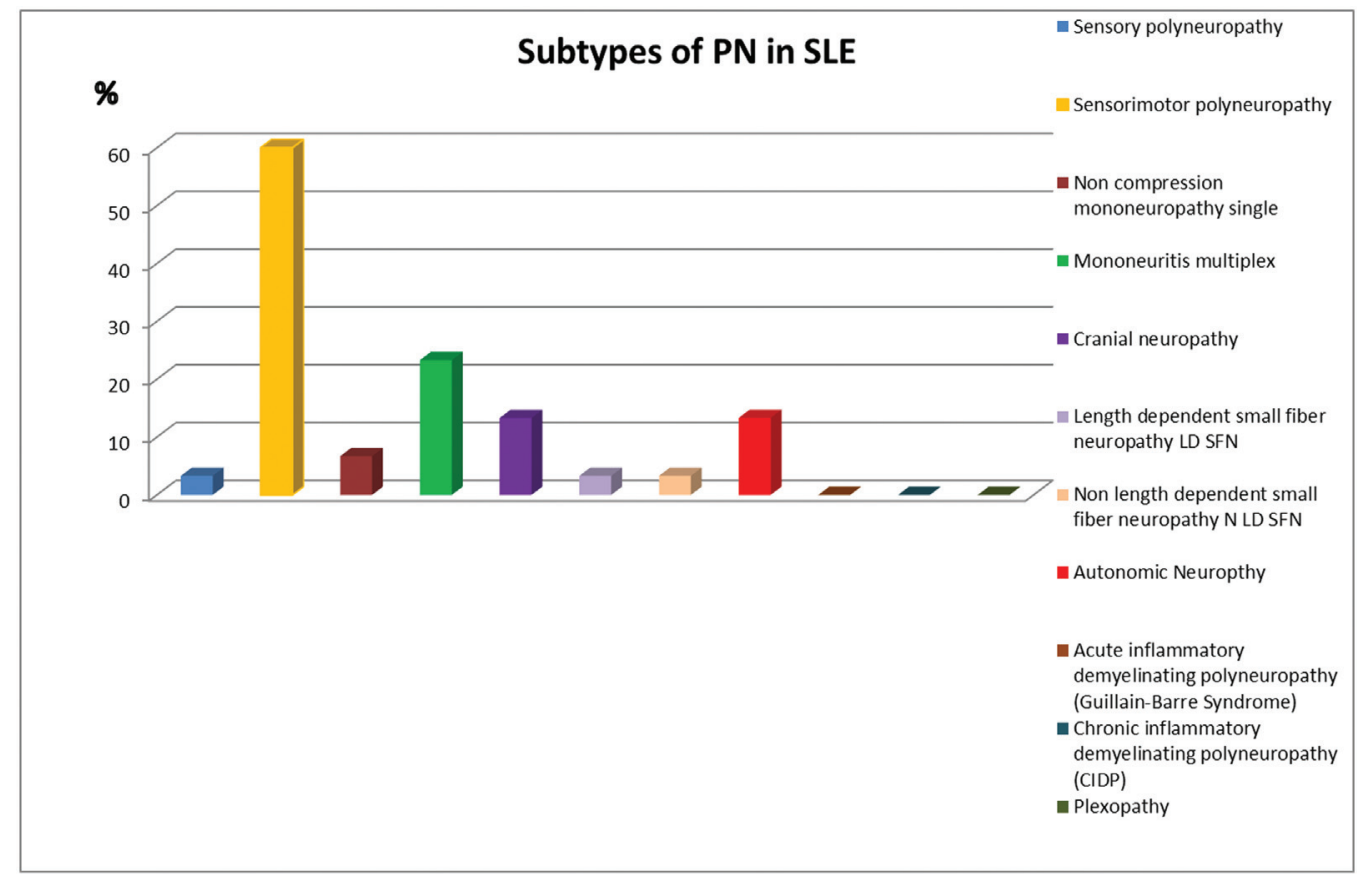

Distribution of the studied cases according to subtypes of PN in group I ( $n=38$ conditions in 30 patients). PN, peripheral neuropathy. 
Comparison between systemic lupus erythematosus with peripheral neuropathy (group I) and systemic lupus erythematosus without peripheral neuropathy (group II) regarding demographic data

There was no statistically significant difference between the two studied groups regarding sex, age, weight, height, and BMI (Table 6 and Figs 4 and 5).

Comparison between systemic lupus erythematosus with peripheral neuropathy (group I) and systemic lupus erythematosus without peripheral neuropathy (group II) regarding disease duration

The disease duration in group I ranged from 3 to 240 months, with median of 36 months. In group I, it ranged from 4 to 168 months, with median of 36 months. There was no statistically significant difference between the two studied groups regarding disease duration (Table 7).

Comparison between systemic lupus erythematosus with peripheral neuropathy (group I) and systemic lupus erythematosus without peripheral neuropathy (group II) regarding systemic lupus erythematosus clinical features

There were no statistically significant differences between the two studied groups [SLE with PN (group I) versus SLE without PN (group II)] regarding SLE clinical features except for malar rash and lupus nephritis.

In group I, 16 (53.3\%) patients had malar rash, in comparison with five $(16.7 \%)$ patients in group I.

Table 2 Distribution of the studied cases according to pathology of peripheral neuropathy in group I $(n=30)$

\begin{tabular}{lc}
\hline Pathology of peripheral neuropathy & $N(\%)$ \\
\hline Axonal & $19(63.3)$ \\
Demyelination & $1(3.33)$ \\
Mixed & $8(26.6)$ \\
Pure SFN (normal sensory motor NC) & $2(6.7)$ \\
\hline
\end{tabular}

$\mathrm{NC}$, nerve conduction; SFN, small fiber neuropathy.
Malar rash showed a significant increase in group I compared with group (Table 8 and Fig. 6).

In group I, 17 (56.7\%) patients had nephritis, in comparison with four (13.3\%) patients in group I. Nephritis showed a significant increase in group I compared with group (Table 8 and Fig. 6).

The most frequent SLE clinical symptom was fatigue, with $30(100 \%)$ patients in group I and 28 (93.3\%) patients in group II.

\section{Comparison between systemic lupus erythematosus} with peripheral neuropathy (group I) and systemic lupus erythematosus without peripheral neuropathy (group II) regarding diseases activity Systemic Lupus Activity Measure index

The diseases activity SLAM index in group I ranged from 7 to 23 , with mean of $12.20 \pm 3.85$. In group I, it ranged from 5 to 14 , with mean of $9.87 \pm 2.26$. The diseases activity SLAM index showed a significant increase in group I compared with group (Table 9 and Fig. 7).

Comparison between systemic lupus erythematosus with peripheral neuropathy (group I) and systemic lupus erythematosus without peripheral neuropathy (group II) regarding acute-phase reactant

The ESR in group I ranged from 15 to 142, with median of $49 \mathrm{~mm} / \mathrm{h}$. In group I, it ranged from 15 to

Table 3 Distribution of the studied cases according to associated entrapments in group I $(n=32$ conditions in 30 patients)

\begin{tabular}{lc}
\hline Associated entrapments & $N(\%)$ \\
\hline No entrapments & $14(46.6)$ \\
CTS & $10(33.3)$ \\
Cubital tunnel & $6(20)$ \\
Ulnar nerve compression at the wrist. & $1(3.3)$ \\
TTS & $1(3.3)$ \\
\hline
\end{tabular}

CTS, carpal tunnel syndrome; TTS, tarsal tunnel syndrome.

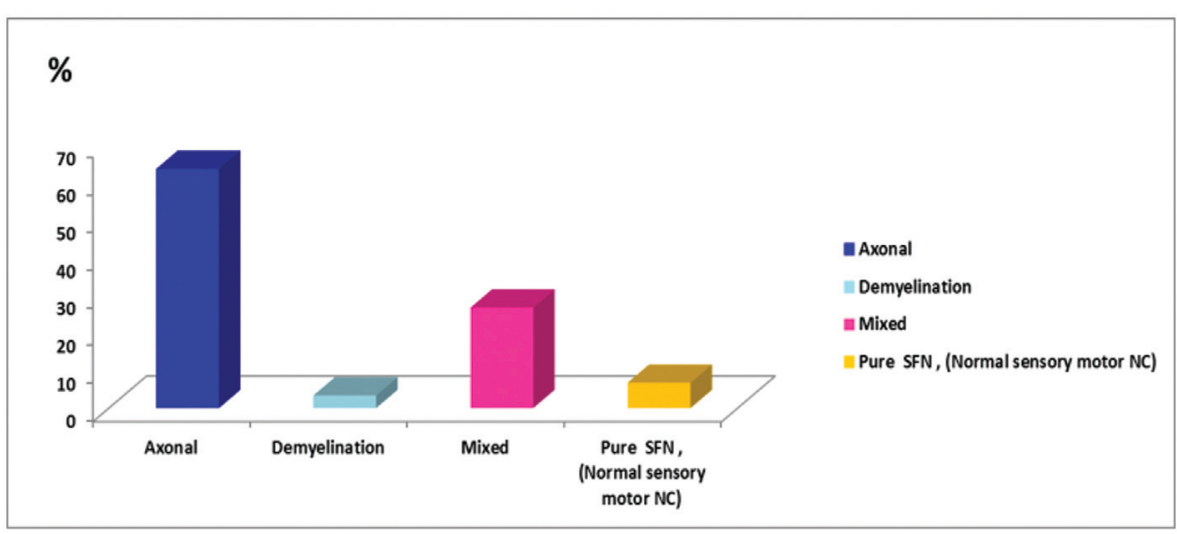

Distribution of the studied cases according to pathology of PN in group I $(n=30)$. PN, peripheral neuropathy. 


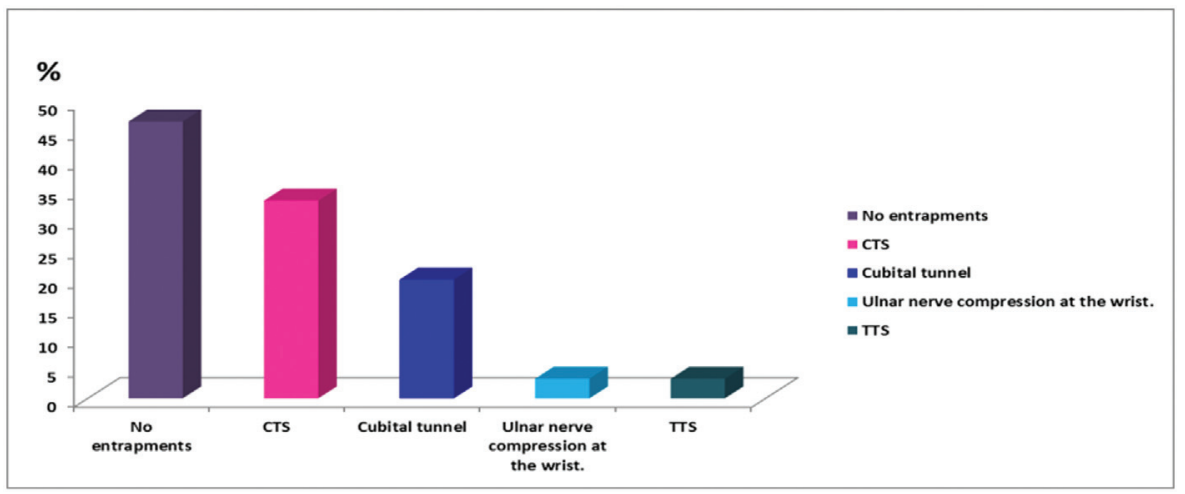

Distribution of the studied cases according to associated entrapments in group I ( $n=32$ conditions in 30 patients). PN, peripheral neuropathy.

130 with median of $41.5 \mathrm{~mm} / \mathrm{h}$. There was no statistically significant difference between the two studied groups according to ESR.

The CRP in group I ranged from 0.90 to 73 with median of $6 \mathrm{mg} / \mathrm{l}$. In group I, it ranged from 2 to 122 with median of $7.5 \mathrm{mg} / \mathrm{l}$. There was no statistically significant difference between the two studied groups regarding CRP (Table 10).

\section{Comparison between systemic lupus erythematosus with peripheral neuropathy (group I) and systemic lupus erythematosus without peripheral neuropathy (group II) regarding immunological markers}

There was no statistically significant difference between the two studied groups regarding ANA, APLs, C3, C4, and anti-dsDNA (Table 11).

\section{Discussion}

The American College of Rheumatology proposed cases definitions and classification criteria for 19 CNS and PNS syndromes observed in SLE [1]. PN is a perceived manifestation of SLE [1-5]. The clinical presentation of PN relies upon the diameter of the affected nerve, the type of demyelinating or axonal damage, and their acute or chronic event [11]. The pathogenesis of SLE-related neuropathy is obscure, and the few pathological studies of the peripheral nerves in SLE have revealed axonal degeneration, inflammatory changes, and vasculitis [13], which may propose the likelihood of various pathogenetic components for the different types of SLE-related neuropathy [14].

The etiopathogenesis of PN relies upon its subtype. Mononeuropathy multiplex develops owing to vasculitic damage to vasa nervosum. Wallerian degeneration of nerve fibers is due to ischemic localized infarction caused by vasculitis, whereas sensory neuropathy is a disorder caused by sensory neurons involvement within the dorsal root ganglion. The degeneration is associated with an inflammatory T-cell reaction driven mainly by a cell-mediated immune response. In fact, nerve biopsy can be invasive and may lead to neurological complications and histological findings can be helpful only in difficult circumstances where nerve conduction studies are noncontributive [11]. If electrodiagnostic studies are normal, small-fiber neuropathy may be diagnosed by quantitative sensory testing [21].

Hardly few studies have found relationship of SLErelated neuropathy with other disease activity and laboratory markers, likely to be owing to small sample size as well as the incorporation of a heterogeneous group of neuropathies in SLE. However, associations have been reported with disease activity and CNS contribution. This case-control study was carried out to investigate electrophysiological patterns of peripheral nerves involvements in patients having SLE in relation to the disease activity.

The results showed that the most common PN subtype is sensorimotor polyneuropathy which occurred in 18 (60\%) patients, and seven (23.3\%) patients had mononeuritis multiplex. Two (6.7\%) patients had cranial neuropathy. Only one (3.33\%) patient had sensory polyneuropathy, two (6.66\%) patients had mononeuropathy (single), one (3.33\%) patient had length-dependent SFN, one (3.33\%) patient had non-length-dependent SFN, and four (13.3\%) patients had autonomic neuropathy. None of the patients had acute inflammatory demyelinating polyneuropathy (Guillain-Barre syndrome) or CIDP or plexopathy. The most common PN pathology was axonal degeneration, which occurred in 19/30 (63.3\%) 
patients, one (1/30) patient (3.33\%) had demyelination, $8 / 30$ (26.6\%) patients had mixed pathology (demyelination and axonal degeneration), and 2/30 (6.7\%) patients had pure SFN with normal peripheral sensory motor nerve conduction study.

Our results were in agreement with those conducted by Toledano et al. [22], Xianbin et al. [23], Saigal et al. [24], Jasmin et al. [13], Salem et al. [11], Oomatia et al. [25], Florica et al. [3], Goh et al., [26] and Omdal et al. $[27,28]$ which found the most frequent PN subtype was sensorimotor polyneuropathy and the most common pattern was the axonal degeneration pathology.

Mccombe et al. [29] investigated the clinical features, nerve conduction studies, and pathological findings in the sural nerves are described in seven patients with $\mathrm{PN}$. The PN was of a chronic sensorimotor type with overwhelmingly sensory manifestations and gradual onset. In two cases, the presentation was asymmetric. One patient had autonomic malfunction. The histopathological characteristics in the biopsied sural nerves were those of axonal degeneration and vasculitis.

A lot of mechanisms have been reported to clarify the peripheral damage in lupus. Neurogenic inflammation is interceded by the release of different neuropeptides such as calcitonin generelated protein, substance $\mathrm{P}$, nitric oxide and chemokines resulting in vasodilatation, increasing vascular porousness and cell trafficking. The major inflammatory mediators released from immune cells act on sensory neurons inducing peripheral sensitization and hyperalgesic phenomena; moreover, after an injury, this natural inflammatory response could facilitate the pathogenetic activity of antineural autoantibodies, in addition to ischemic vascular mechanism by vasa nervorum vascularitis or by microthrombi linked to antiphospholipid antibodies. The other legitimate mechanisms are: immunologic effect by a direct antibodies aggression, entraining destruction of the peripheral nerve component [15-17].

In this study, only one patient showed evidence of subclinical neuropathy. In contradiction to this result, Goh et al. [26] stated that the subclinical $\mathrm{PN}$ is common in patients with SLE. Moreover, Fathalla and El-Badawy [30] investigated the association of subclinical autonomic and PN with SLE. Pure sensory, mixed sensory-motor abnormalities were detected in two patients. SSR 


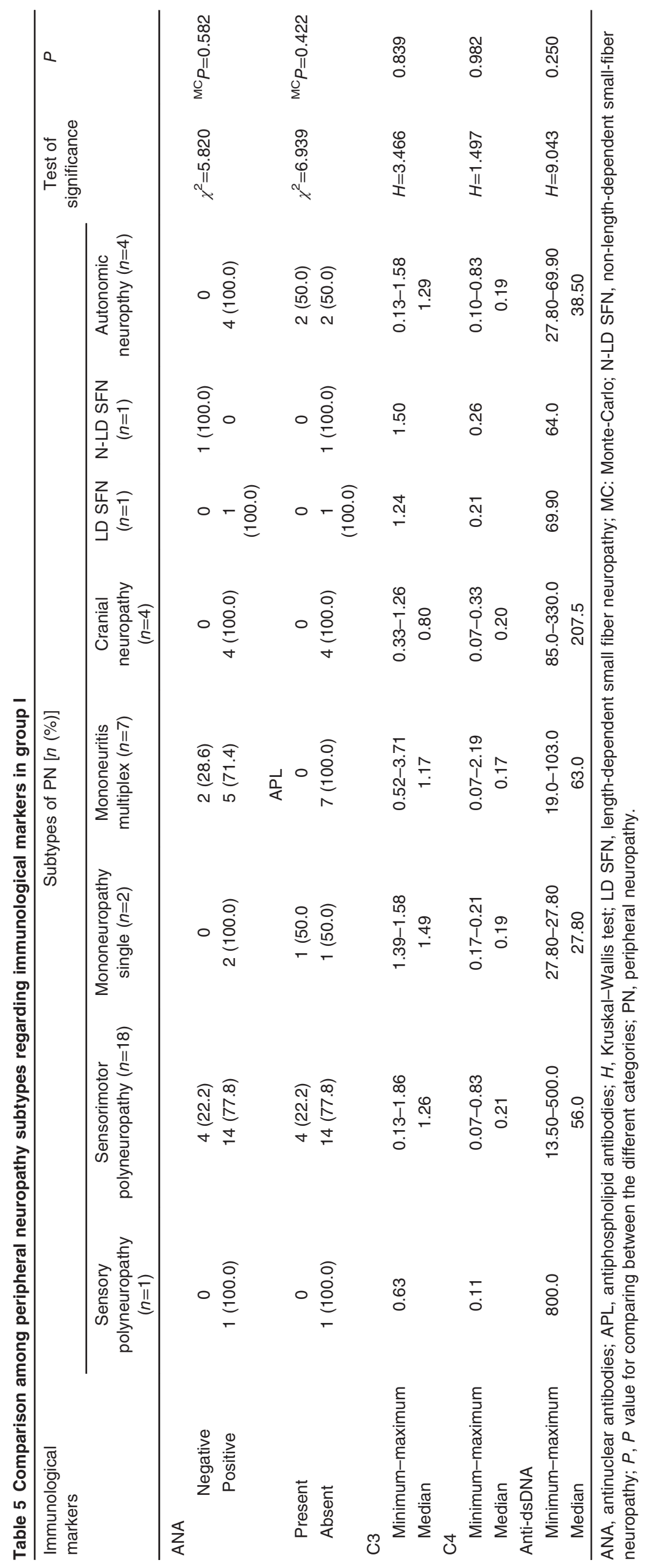


Table 6 Comparison between systemic lupus erythematosus with peripheral neuropathy (group I) and systemic lupus erythematosus without peripheral neuropathy (group II) regarding demographic data

\begin{tabular}{|c|c|c|c|c|}
\hline & Group I SLE with PN $(n=30)[n(\%)]$ & Group II SLE without PN $(n=30)[n(\%)]$ & Test of significance & $P$ \\
\hline \multicolumn{5}{|l|}{ Sex } \\
\hline Female & $26(86.7)$ & $27(90.0)$ & $\chi^{2}=0.162$ & ${ }^{\mathrm{FE}} P=1.000$ \\
\hline Male & $4(13.3)$ & $3(10.0)$ & & \\
\hline \multicolumn{5}{|l|}{ Age (years) } \\
\hline Range & $18.0-55.0$ & $18.0-58.0$ & $t=0.449$ & 0.655 \\
\hline Mean \pm SD & $37.43 \pm 9.34$ & $36.30 \pm 10.19$ & & \\
\hline \multicolumn{5}{|l|}{ Weight (kg) } \\
\hline Range. & $40.0-90.0$ & $49.0-91.0$ & $t=1.457$ & 0.151 \\
\hline Mean \pm SD & $70.20 \pm 11.33$ & $74.40 \pm 11.0$ & & \\
\hline \multicolumn{5}{|l|}{ Height (m) } \\
\hline Range. & $1.50-1.76$ & $1.55-1.76$ & $t=0.522$ & 0.604 \\
\hline Mean \pm SD & $1.63 \pm 0.06$ & $1.64 \pm 0.06$ & & \\
\hline \multicolumn{5}{|l|}{ BMI $\left(\mathrm{kg} / \mathrm{m}^{2}\right)$} \\
\hline Range & $16.60-33.20$ & $19.91-35.41$ & $t=1.469$ & 0.147 \\
\hline Mean \pm SD & $26.33 \pm 4.21$ & $27.91 \pm 4.13$ & & \\
\hline
\end{tabular}

FE, Fisher exact; $P, P$ value for comparing from the two groups; PN, peripheral neuropathy; SLE, systemic lupus erythematosus; $t$, Student $t$-test.

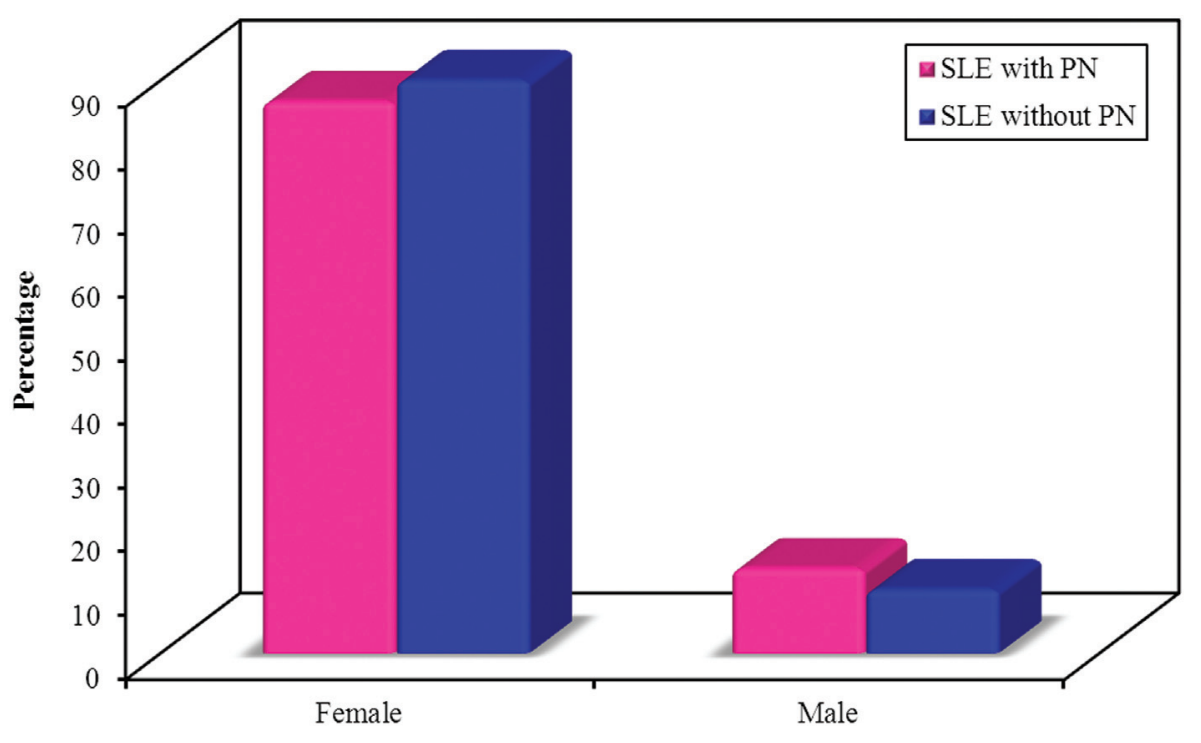

Comparison between SLE with PN (group I) and SLE without PN (group II) regarding sex. PN, peripheral neuropathy; SLE, systemic lupus erythematosus.

was not elicited in 13 of 43 patients, whereas latency and amplitude abnormalities were detected in 11 of 43 and 9 of 43 patients, respectively. The sympathetic nervous system was affected in up to $40 \%$ of patients with lupus according to the study by Fathalla and ElBadawy [30].

This study did not show that frequency of subclinical PN in patients with SLE and the percentage of sympathetic nervous system affection was modest [four (13.3\%) patients] in comparison with $40 \%$ in the study by Fathalla and El-Badawy [30]. The possible explanation is that the diagnosis of sympathetic neuropathy should be confirmed by the ACR nomenclature and case definitions for neuropsychiatric lupus syndromes after the screening by single diagnostic tool such as SSR.

Regarding common entrapment neuropathies, 10 (33.3\%) patients had CTS, six (20\%) patients had cubital tunnel syndrome, one (3.3\%) patient had ulnar nerve compression at the wrist, one (3.3\%) patient had tarsal tunnel syndrome, whereas 14 (46.66\%) patients showed no evidence of entrapments. The only studied entrapment neuropathy was the carpal tunnel syndrome 


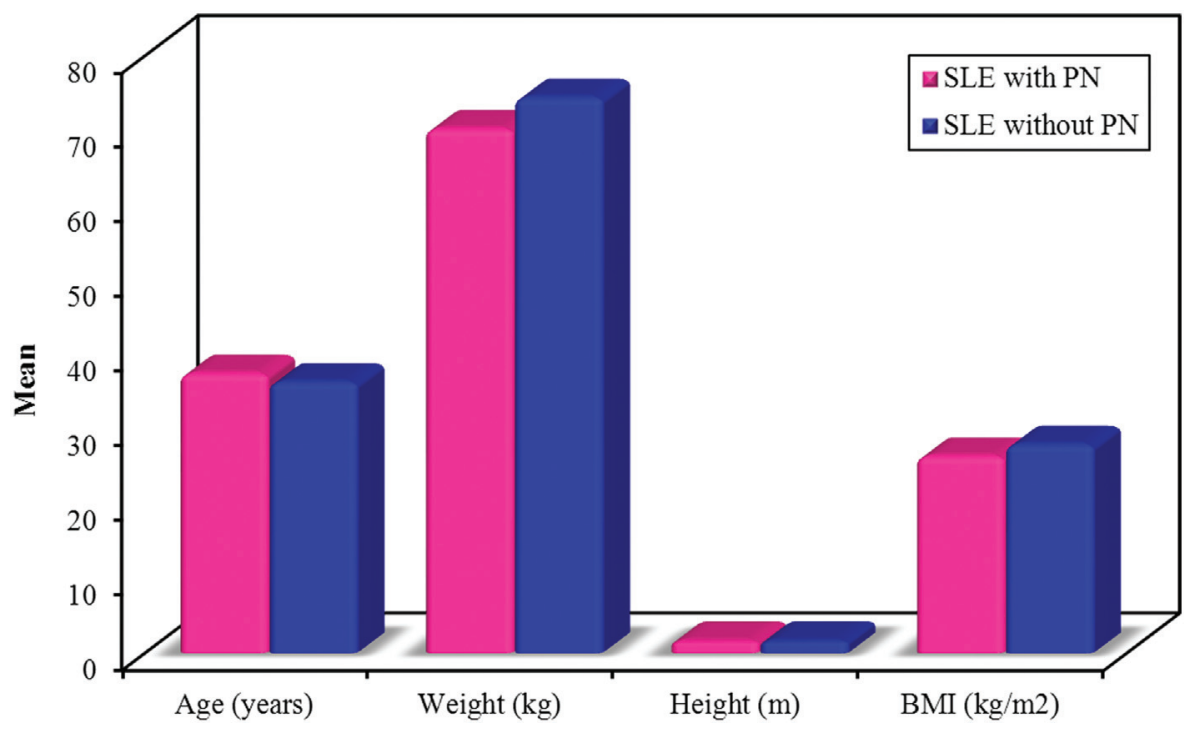

Comparison between SLE with PN (group I) and SLE without PN (group II) regarding age (years), weight $(\mathrm{kg})$, height $(\mathrm{m})$, and BMI $\left(\mathrm{kg} / \mathrm{m}^{2}\right)$. PN, peripheral neuropathy; SLE, systemic lupus erythematosus.

Table 7 Comparison between systemic lupus erythematosus with peripheral neuropathy (group I) and systemic lupus erythematosus without peripheral neuropathy (group II) regarding disease duration

\begin{tabular}{|c|c|c|c|c|}
\hline & $\begin{array}{c}\text { SLE with } \\
\text { PN } \\
(n=30)\end{array}$ & $\begin{array}{c}\text { SLE } \\
\text { without PN } \\
(n=30)\end{array}$ & $U$ & $P$ \\
\hline \multicolumn{5}{|c|}{ Diseases duration (months) } \\
\hline Minimum-maximum & $3.0-240.0$ & $4.0-168.0$ & 423.50 & 0.694 \\
\hline Median & 36.0 & 36.0 & & \\
\hline
\end{tabular}

$U$, Mann-Whitney test; $P, P$ value for comparing from the two groups; PN, peripheral neuropathy; SLE, systemic lupus erythematosus.

described by Jasmin et al. [13] and Toledano et al. [22]. Jasmin et al. [13] reported a total of 17 (11.5\%) patients (nine bilateral and eight unilateral) had NCS evidence of median nerve conduction slowing at the wrists indicative of CTS; in eight (5.4\%) of whom, these were the only NCS abnormalities. The prevalence of carpal tunnel syndrome in the study conducted by Toledano et al. [22] was $4.2 \%$, and it is like that found in the general population (3.8-4.9\%), thus proposing that SLE is not, probably, a direct cause of this compression syndrome in most cases. The different prevalence among studies can be clarified by different CTS risk factors and socioeconomic state. Risk factors were being female, being middleaged, having a high BMI, smoking, and low socioeconomic status [31].

In this study, in comparison between group I (SLE with PN) and group II, there was no statistically significant difference between the two groups regarding demographic data, disease duration, and lupus clinical features except malar rash and lupus nephritis, which showed significant increase in group I compared with group II $(P=0.003$ and $P<0.001$, respectively).

In agreement with the current study, Florica et al. [3] reported no significant difference between SLE-related PN and non-SLE-related PN group regarding demographic and clinical information. However, patients with SLE-related PN had significantly shorter SLE disease duration and tended to be younger in contrast with those with non-SLE-related PN, whereas Jasmin et al. [13] stated that SLE-related polyneuropathy patients were significantly older (mean age 44.7 vs. 37.5 years), but had no other significant demographic or disease associations, in contrast with non-SLE-related neuropathy.

Oomatia et al. [25] compared between patients with SLE with PN and patients with SLE without PN, and in contrast to this study, those with $\mathrm{PNs}$ were more likely to have a history of infections $(P<0.01)$ as well as osteoporotic fractures $(P<0.01)$. There was no significant difference between the two studied groups regarding other clinical manifestations (malar rash and nephritis), and demographic data. In contrast, Saigal et al. [24] reported significant association of PN in patients with SLE with pyuria, pleurisy, and leucopenia, whereas Toledano et al. [22] stated that the involvement of PN occurs more frequently in patients who are diagnosed with SLE at older age and showed relation of hematological and renal manifestations in SLE with PN group. 
Table 8 Comparison between systemic lupus erythematosus with peripheral neuropathy (group I) and systemic lupus erythematosus without peripheral neuropathy (group II) regarding systemic lupus erythematosus clinical features

\begin{tabular}{|c|c|c|c|c|}
\hline Clinical features & SLE with PN $(n=30)[n(\%)]$ & SLE without PN $(n=30)[n(\%)]$ & $x^{2}$ & $p$ \\
\hline Fever & $11(36.7)$ & $6(20.0)$ & 2.052 & ${ }^{\mathrm{FE}} P=0.152$ \\
\hline Fatigue & $30(100.0)$ & $28(93.3)$ & 2.069 & ${ }^{\mathrm{FE}} P=0.492$ \\
\hline Malar rash & $16(53.3)$ & $5(16.7)$ & $8.864^{*}$ & ${ }^{\mathrm{FE}} P=0.003^{*}$ \\
\hline Chronic cutaneous & $4(13.3)$ & $6(20.0)$ & 0.480 & ${ }^{\mathrm{FE}} P=0.488$ \\
\hline Photosensitivity & $27(90.0)$ & $22(73.3)$ & 2.783 & ${ }^{\mathrm{FE}} P=0.095$ \\
\hline Alopecia & $24(80.0)$ & $27(90.0)$ & 1.176 & ${ }^{\mathrm{FE}} P=0.472$ \\
\hline Oral ulcers & $21(70.0)$ & $22(73.3)$ & 0.082 & ${ }^{\mathrm{FE}} P=0.774$ \\
\hline Arthralgia/ arthritis & $29(96.7)$ & $26(86.7)$ & 1.964 & ${ }^{\mathrm{FE}} P=0.353$ \\
\hline Raynaud's phenomenon & $3(10.0)$ & $2(6.7)$ & 0.218 & ${ }^{\mathrm{FE}} P=1.000$ \\
\hline Vasculitis & $5(16.7)$ & $3(10.0)$ & 0.577 & ${ }^{\mathrm{FE}} P=0.706$ \\
\hline Serositis & $3(10.0)$ & $4(13.3)$ & 0.162 & ${ }^{\mathrm{FE}} P=1.000$ \\
\hline Nephritis & $17(56.7)$ & 4 (13.3) & $12.381^{*}$ & ${ }^{\mathrm{FE}} P \leq 0.001^{*}$ \\
\hline Pneumonia & $3(10.0)$ & $4(13.3)$ & 0.162 & ${ }^{\mathrm{FE}} P=1.000$ \\
\hline \multicolumn{5}{|l|}{ CNS } \\
\hline Headache & $12(40.0)$ & $9(30.0)$ & 0.659 & ${ }^{\mathrm{FE}} P=0.417$ \\
\hline Seizures & $2(6.7)$ & 0 & 2.069 & ${ }^{\mathrm{FE}} P=0.492$ \\
\hline Psychosis & $2(6.7)$ & $2(6.7)$ & 0.000 & ${ }^{\mathrm{FE}} P=1.000$ \\
\hline Cognitive deficit & 0 & $2(6.7)$ & 2.069 & ${ }^{\mathrm{FE}} P=0.492$ \\
\hline CVS & $2(6.7)$ & $1(3.3)$ & 0.351 & ${ }^{\mathrm{FE}} P=1.000$ \\
\hline Transverse myelitis & $2(6.7)$ & 0 & 2.069 & ${ }^{\mathrm{FE}} P=0.492$ \\
\hline \multicolumn{5}{|l|}{ Hematological disease } \\
\hline Anemia & $16(53.3)$ & $18(60.0)$ & 0.271 & ${ }^{\mathrm{FE}} P=0.602$ \\
\hline Leukopenia & $6(20.0)$ & $6(20.0)$ & 0.000 & ${ }^{\mathrm{FE}} P=1.000$ \\
\hline Thrombocytopenia & $4(13.3)$ & $3(10.0)$ & 0.162 & ${ }^{\mathrm{FE}} P=1.000$ \\
\hline Pancytopenia & $3(10.0)$ & 0 & 3.158 & ${ }^{\mathrm{FE}} P=0.237$ \\
\hline Thrombosis & $5(16.7)$ & $3(10.0)$ & 0.577 & ${ }^{\mathrm{FE}} P=0.706$ \\
\hline Hepatosplenomegaly & $5(16.7)$ & $1(3.3)$ & 2.963 & ${ }^{\mathrm{FE}} P=0.195$ \\
\hline Lymphadenopathy & $4(13.3)$ & $5(16.7)$ & 0.131 & ${ }^{\mathrm{FE}} P=1.000$ \\
\hline Osteoporosis & $3(10.0)$ & $1(3.3)$ & 1.071 & ${ }^{\mathrm{FE}} P=0.612$ \\
\hline Avascular necrosis & $1(3.3)$ & 0 & 1.017 & ${ }^{\mathrm{FE}} P=1.000$ \\
\hline
\end{tabular}

CVS, cerebrovascular stroke; $P, P$ value for comparing from the two groups; PN, peripheral neuropathy; SLE, systemic lupus erythematosus. ${ }^{*} P \leq 0.05$, statistically significant.

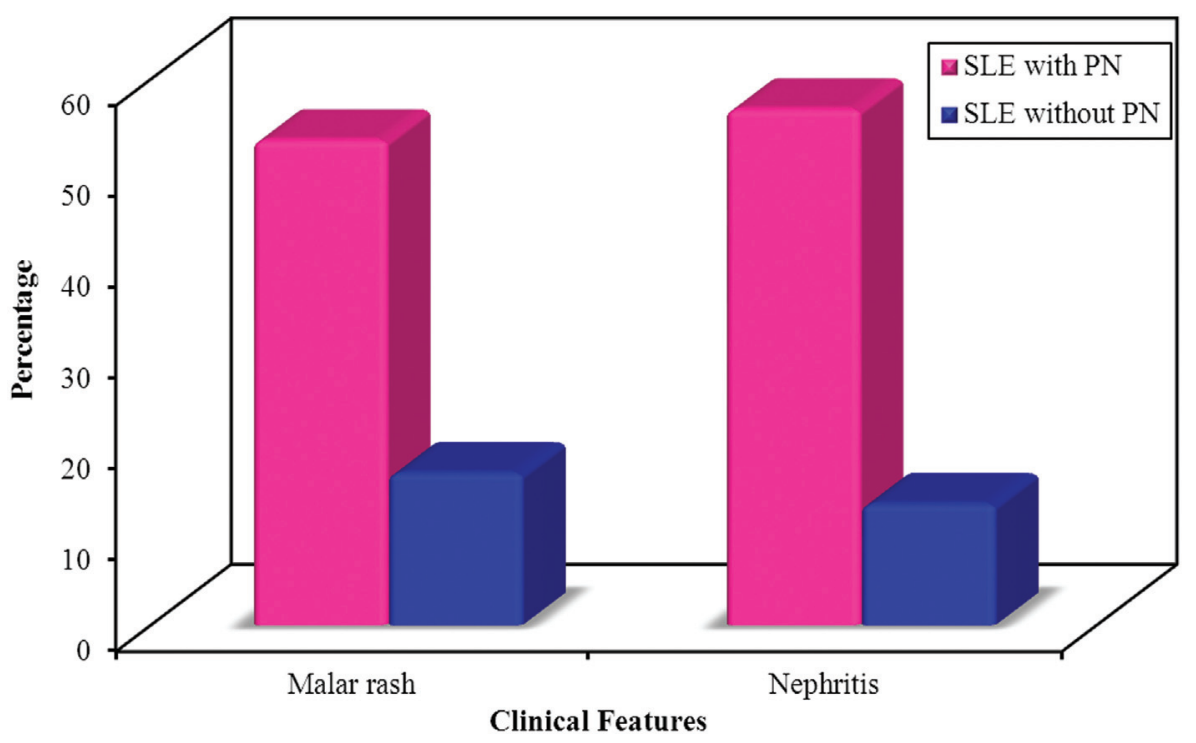

Comparison between SLE with PN (group I) and SLE without PN (group II) regarding SLE clinical features (malar rash and nephritis). PN, peripheral neuropathy; SLE, systemic lupus erythematosus. 
Xianbin et al. [23] expressed that patients with SLE with PN had a greater frequency of fever (65.8 vs. 45.9\%, $P<0.01)$, mucocutaneous lesions (73.9 vs. $36.3 \%, \quad P<0.01)$, arthritic involvement $(42.5$ vs. $28.1 \%, P<0.05)$, myositis (17.8 vs. $5.5 \%, P<0.01$ ), and CNS contribution (38.4 vs. $21.9 \%, P<0.05)$ compared with non-SLE-PN group.Regarding diseases activity in this study, SLAM index showed a significant increase in group I compared with group $(P=0.006)$. Florica et al. [3] revealed that patients with SLE-related PN had an essentially higher median systemic lupus erythematosus Disease Activity Index (SLEDAI)-2K than those with non-SLE-related PN. In concordance, Saigal et al. [24] suggested significant association of $\mathrm{PN}$ in patients with SLE with modified SLEDAI-2000, which is a marker of disease activity and chronic inflammation, clarifying its relationship with PN in this study. In addition, Xianbin et al. [23] revealed statistically significant difference of SLEDAI scores in patients with SLE-PN in contrast with the non-SLE-PN group $(P<0.05)$, whereas Oomatia et al.

Table 9 Comparison between systemic lupus erythematosus with peripheral neuropathy (group I) and systemic lupus erythematosus without peripheral neuropathy (group II) regarding diseases activity Systemic Lupus Activity Measure index

\begin{tabular}{lcccc}
\hline Diseases activity & SLE with & SLE without & $t$ & $p$ \\
SLAM index & PN $(n=30)$ & PN $(n=30)$ & & \\
\hline Range & $7.0-23.0$ & $5.0-14.0$ & $2.862^{*}$ & $0.006^{*}$ \\
Mean \pm SD & $12.20 \pm 3.85$ & $9.87 \pm 2.26$ & & \\
\hline
\end{tabular}

$P, P$ value for comparing from the two groups; $P N$, peripheral neuropathy; SLAM, Systemic Lupus Activity Measure; SLE, systemic lupus erythematosus. ${ }^{*} P \leq 0.05$, statistically significant.
[25] demonstrated that SLE with PNs had lower disease activity $(P=0.01)$ and higher disease damage $(P<0.01)$ in contrast with SLE group without PN.

In contradiction, Jasmin et al. [13] stated that most patients with SLE with PN did not have active disease (median SLEDAI=2) at the time of the study and no significant contributions of polyneuropathy were observed with current disease activity, number of relapses, or previous severe disease manifestations. However, this does not exclude the activity of lupus disease at the onset of neuropathy; its long-term persistence did not appear to be related to active or severe disease. This could mirror the nature of an axonal neuropathy, in which recovery from axonal denervation is usually deficient resulting in a chronic and persistent deficit. Consequently, contrasts in

Table 10 Comparison between systemic lupus erythematosus with peripheral neuropathy (group I) and systemic lupus erythematosus without peripheral neuropathy (group II) regarding acute-phase reactant

\begin{tabular}{lcccc}
\hline Acute-phase reactant & SLE with & SLE without & $U$ & $P$ \\
& PN $(n=30)$ & PN $(n=30)$ & & \\
\hline ESR first hour & & & & \\
$\quad$ Minimum-maximum & $15.0-142.0$ & $15.0-130.0$ & 400.0 & 0.460 \\
$\quad$ Median & 49.0 & 41.50 & & \\
CRP & & & & \\
$\quad$ Minimum-maximum & $0.90-73.0$ & $2.0-122.0$ & 401.0 & 0.468 \\
$\quad$ Median & 6.0 & 7.50 & & \\
\hline
\end{tabular}

CRP, C-reactive protein; ESR, erythrocytes sedimentation rate; $P$, $P$ value for comparing from the two groups; $P N$, peripheral neuropathy; SLE, systemic lupus erythematosus; $U$, Mann-Whitney test.

\section{Figure 7}

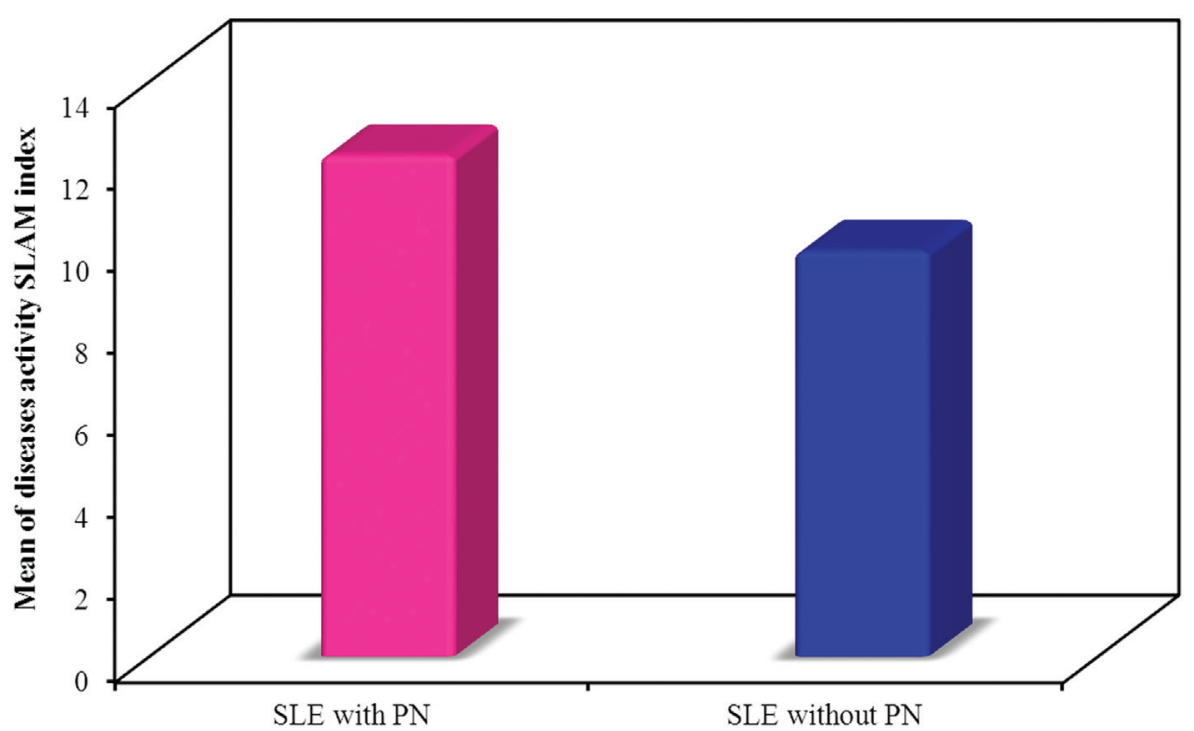

Comparison between SLE with PN (group I) and SLE without PN (group II) regarding diseases activity Systemic Lupus Activity Measure (SLAM) index. PN, peripheral neuropathy; SLE, systemic lupus erythematosus. 
Table 11 Comparison between systemic lupus erythematosus with peripheral neuropathy (group I) and systemic lupus erythematosus without peripheral neuropathy (group II) regarding immunological markers

\begin{tabular}{|c|c|c|c|c|}
\hline Immunological markers & SLE with PN $(n=30)[n(\%)]$ & SLE without PN $(n=30)[n(\%)]$ & Test of significance & $P$ \\
\hline \multicolumn{5}{|l|}{ ANA } \\
\hline Negative & $7(23.3)$ & $7(23.3)$ & $\chi^{2}=0.000$ & 1.000 \\
\hline Positive & $23(76.7)$ & $23(76.7)$ & & \\
\hline \multicolumn{5}{|l|}{ APL } \\
\hline Present & $5(16.7)$ & $3(10.0)$ & $\chi^{2}=0.577$ & ${ }^{\mathrm{FE}} P=0.706$ \\
\hline Absent & 25 (83.3) & $27(90.0)$ & & \\
\hline \multicolumn{5}{|l|}{ C3 } \\
\hline Minimum-maximum & $0.13-3.71$ & $0.31-2.60$ & $U=424.0$ & 0.701 \\
\hline Median & 1.28 & 1.21 & & \\
\hline \multicolumn{5}{|l|}{$\mathrm{C} 4$} \\
\hline Minimum-maximum & $0.07-10.50$ & $0.08-1.29$ & $U=432.50$ & 0.796 \\
\hline Median & 0.21 & 0.18 & & \\
\hline \multicolumn{5}{|l|}{ Anti-dsDNA } \\
\hline Minimum-maximum & $13.50-800.0$ & $15.0-669.0$ & $U=421.0$ & 0.668 \\
\hline Median & 56.0 & 47.65 & & \\
\hline
\end{tabular}

FE, Fisher exact; $U$, Mann-Whitney test; $P, P$ value for comparing from the two groups; PN, peripheral neuropathy; SLE, systemic lupus erythematosus.

disease activity may account for differences in the frequency and spectrum of PNs.

In this study, acute-phase reactant (ESR and CRP) and immunological markers (ANA, antiphospholipid antibodies (APL), C3, C4, and anti-dsDNA) showed no statistically significant difference between SLE with $\mathrm{PN}$ and SLE without PN groups. These results were in agreement with Florica et al. [3], Jasmin et al. [13], and Oomatia et al. [25], which all showed no statistically significant difference between SLE with PN compared with SLE non-related PN or SLE without PN groups. In contrast to the current results, Xianbin et al. [23] revealed that patients with SLE-PN had low serum C3 level (54.8 vs. $36.9 \%, P<0.05)$, whereas no significant difference between the two groups with respect to ANA, anti-dsDNA, and ESR. Saigal et al. [24] proposed significant association of PN in SLE patients with ESR level. These variations can be explained by the same theory of chronicity; it does not exclude disease being active at the onset of polyneuropathy; its persistence did not seem to be contributed to disease activity or severity. This could reflect the nature of an axonal neuropathy, in which recovery from axonal denervation is usually deficient resulting in a chronic disorder.

\section{Conclusion}

(1) PN is common in patients with SLE and sensorymotor polyneuropathy is the most frequent subtype.

(2) The pathology of PN in SLE is mainly axonal.
(3) PN in patients with SLE is related to disease activity, lupus nephritis, and skin involvement.

\section{Financial support and sponsorship}

Nil.

\section{Conflicts of interest}

There are no conflicts of interest.

\section{References}

1 [No authors listed]. The American College of Rheumatology nomenclature and case definitions for neuropsychiatric lupus syndromes. Arthritis Rheum 1999; 42:599-608.

2 Brey RL, Holiday SL, Saklad AR, Navarrete MG, Hermosillo RD, Stallworth $\mathrm{CL}$, et al. Neuropsychiatric syndromes in lupus: prevalence using standardized definitions. Neurology 2002; 58:1214-1220.

3 Florica B, Aghdassi E, Jiandong S, Dafna D, Murray B, Paul R, et al. Peripheral neuropathy in patients with systemic lupus erythematosus. Semin Arthritis Rheum 2011; 41:203-211.

4 Mok C, Lau S, Wong R. Neuropsychiatric manifestations and their clinical associations in southern Chinese patients with systemic lupus erythematosus. J Rheumatol 2001; 28:766-771.

5 Bertsias GK, loannidis JP, Aringer M, Bollen E, Bombardieri S, Bruce IN, et al. EULAR recommendations for the management of systemic lupus erythematosus with neuropsychiatric manifestations: report of a task force of the EULAR standing committee for clinical affairs. Ann Rheum Dis 2010; 69:2074-2082.

6 Hanly JG, Urowitz MB, Su L, Bae SC, Gordon C, Wallace DJ, et al. Prospective analysis of neuropsychiatric events in an international disease inception cohort of patients with systemic lupus erythematosus. Ann Rheum Dis 2010; 69:529-535.

7 Vina ER, Fang AJ, Wallace DJ, Weisman MH. Chronic inflammatory demyelinating polyneuropathy in patients with systemic lupus erythematosus: prognosis and outcome. Semin Arthritis Rheum 2005 35:175-184.

8 Goh KJ. Successful treatment of chronic inflammatory demyelinating polyneuropathy in systemic lupus erythematosus with oral cyclophosphamide. Lupus 2012; 21:1119-1123.

9 Shoshtary J, Adib M. Peripheral neuropathy in systemic lupus erythematosus in Southern Iran. Electromyogr Clin Neurophysiol 2005; 45:145-148.

10 Harboe E, Tjensvoll AB, Maroni S, Gøransson LG, Greve OJ, Beyer MK, et al. Neuropsychiatric syndromes in patients with systemic lupus 
erythematosus and primary Sjögren syndrome: a comparative populationbased study. Ann Rheum Dis 2009; 68:1541-1546.

11 Salem TB, Belfeki N, Lamloum M, Khanfir M, Ben Ghorbel I, Houman MH. Peripheral neuropathies in patients with systemic lupus erythematosus. J Neurol Neurosci 2016; 7:S3.

12 Hoitsma E, Reulen JP, de Baets M, Drent M, Spaans F, Faber CG. Small fiber neuropathy: a common and important clinical disorder. J Neurol Sci 2004; 227:119-130.

13 Jasmin R, Sockalingam S, Ramanaidu LP, Goh KJ. Clinical and electrophysiological characteristics of symmetric polyneuropathy in a cohort of systemic lupus erythematosus patients. Lupus 2015; 24:248-255.

14 Ramos-Casals M, Nardi N, Lagrutta M, Brito-Zerón P, Bové A, Delgado G, et al. Vasculitis in systemic lupus erythematosus: prevalence and clinical characteristics in 670 patients. Medicine (Baltimore) 2006; 85:95-104.

15 Yoshio T, Okamoto H. Pathogenesis of neuropsychiatric syndromes of systemic lupus erythematosus. Open J Rheumatol Autoimmune Dis 2015; 5:46-56.

16 Jeltsch-David H, Muller S. Neuropsychiatric systemic lupus erythematosus: pathogenesis and biomarkers. Nat Rev Neurol 2014; 10:579-596.

17 Bortoluzzi A, Silvagni E, Furini F, Piga M, Govoni M. Peripheral nervous system involvement in systemic lupus erythematosus: a review of the evidence. Clin Exp Rheumatol; 2019; 37:146-155.

18 Navinan M, Piranavan P, Uthuman A, Akram A, Yudhishdran J, Kandeepan T. Sensory neuronopathy complicating systemic lupus erythematosus: a case report. J Med Case Rep 2014; 8:141

19 Petri M, Orbai AM, Alarcon GS, Gordon C, Merrill JT, Fortin PR, et al. Derivation and validation of the Systemic Lupus International Collaborating Clinics classification criteria for systemic lupus erythematosus. Arthritis Rheum 2012; 64:2677-2686.

20 Uddin Z, Macdermid J, Packham T. Ice-water (cold stress) immersion testing. J Physiother 2013; 59:277.

21 Devigili G, Tugnoli V, Penza P, Camozzi F, Lombardi R, Melli G, et al. The diagnostic criteria for small fibre neuropathy: from symptoms to neuropathology. Brain 2008; 131:1912-1925.
22 Toledano P, Orueta R, Rodríguez-Pintó I, Valls-Solé J, Cervera R, Espinosa G. Peripheral nervous system involvement in systemic lupus erythematosus: prevalence, clinical and immunological characteristics, treatment and outcome of a large cohort from a single centre. Autoimmun Rev 2017; 16:750-755.

23 Xianbin W, Mingyu W, Dong X, Huiying L, Yan X, Fengchun Z, et al. Peripheral neuropathies due to systemic lupus erythematosus in China. Medicine (Baltimore) 2015; 94:e625.

24 Saigal R, Bhargav R, Goyal L, Agrawal A, Mital P, Wadhwani D. Peripheral neuropathy in systemic lupus erythematosus: clinical and electrophysiological properties and their association with disease activity parameters. J Assoc Physicians India 2015; 63:15-19.

25 Oomatia A, Fang H, Petri M, Birnbaum J. Peripheral neuropathies in systemic lupus erythematosus: clinical features, disease associations, and immunologic characteristics evaluated over a twenty-five-year study period. Arthritis Rheumatol 2014; 66:1000-1009.

26 Goh KJ, Wang CL, Tan CT. Peripheral neuropathy in SLE electrophysiological features in 50 consecutive patients. Neurol J Southeast Asia 1996; 1:47-51.

27 Omdal R, Loseth S, Torbergsen T, Koldingsnes W, Husby G, Mellgren S. Peripheral neuropathy in systemic lupus erythematosus a longitudinal study. Acta Neurol Scand 2001; 103:386-391.

28 Omdal R, Mellgren SI, Husby G, Salvesen R, Henriksen OA, Torbergsen T. A controlled study of peripheral neuropathy in systemic lupus erythematosus. Acta Neurol Scand $1993 ; 88: 41-46$.

29 McCombe PA, McLeod JG, Pollard JD, Guo YP, Ingall TJ. Peripheral sensorimotor and autonomic neuropathy associated with systemic lupus erythematosus. Clinical, pathological and immunological features. Brain 1987; 110:533-549.

30 Fathalla MM, El-Badawy MA. Upper extremity subclinical autonomic and peripheral neuropathy in systemic lupus erythematosus. Egypt Rheumatol Rehabil 2015; 42:87-93.

31 Lee HJ, Lim HS, Kim HS. Socioeconomic status associated with carpal tunnel syndrome: a retrospective nationwide 11-year population-based cohort study in South Korea. bioRxiv 2018; 253633. 